\title{
Acceso y usos de internet en el occidente de México: el caso de Jalisco
}

Este trabajo representa una aproximación inicial a la presencia de la internet en una región de México. El estudio muestra la penetración de la red y los usos más recurrentes entre los internautas del estado de Jalisco. El fenómeno se analiza en el contexto de las tendencias más amplias de "consumo mediático" que imperan en la entidad. El análisis se deriva de los resultados de una encuesta, elaborada en 2006, que evalúa el peso de los medios de comunicación, los usos y las preferencias de las audiencias en ese estado de la república mexicana.

PALABRAS CLAVE: internet, audiencias, consumo mediático, México.
This article synthesizes the findings of a survey on internet and mass media consumption carried out in Jalisco, a state located in western Mexico. It describes and discusses the access and uses of the web among different sectors of the population and evaluates the extent to which the phenomenon of "digital divided" is present in this particular context. The essay also brings into consideration the differences of media consumption patterns between those who have access to internet and those who are so far excluded from this.

KEY WORDS: internet, audiences, media consumption, Mexico.

1 Universidad de Guadalajara.

Correo electrónico: ramales52@yahoo.com.mx 
Poco más de una década después de la instauración de internet como un medio de amplio acceso (con el desarrollo de los llamados browsers o navegadores), la red sigue un incontenible proceso de penetración en todos los rincones de la aldea global. Sus diversos usos y aplicaciones permean lo mismo la economía y la política que el entretenimiento, las expresiones culturales y las formas de interacción social. De la mano de otros avances tecnológicos en el área de las telecomunicaciones y la informática, internet se vuelve accesible a través de distintos soportes, al tiempo que se consolida como un "medio de medios", un espacio de confluencia para los medios de comunicación tradicionales (televisión, radio, cine y prensa) que buscan en este nicho nuevos derroteros para su desarrollo y, en algunos casos, la supervivencia.

El crecimiento de la red es tal, que en términos estadísticos las cifras que dibujan el avance de este medio de comunicación siempre resultan provisionales y en el misma medida efímeras. Día tras día el alcance de la red se expande sigilosamente en todas las latitudes y va delineando la geografía de una sociedad caracterizada por la conectividad digital (Castells, 2003).

Al concluir el año 2005 el número de internautas en el mundo sumaba casi mil millones de mujeres y hombres, de los cuales $28.6 \%$ se localizaba en el continente americano, $38.2 \%$ en Asia, $27.95 \%$ en Europa, $3.39 \%$ en África y $1.76 \%$ en Oceanía (ITU, 2005). Fuentes alternativas indican que el crecimiento de usuarios de internet entre el año 2000 y 2005, a nivel mundial, alcanzó 189\%. Cabe aclarar que esta es una tendencia que refleja situaciones más relativas que absolutas. Con todo, el incremento porcentual de internautas en ciertas regiones del planeta durante este lustro no deja de llamar la atención. En África y Oriente Medio rebasó el nivel de 400 puntos porcentuales, en otros casos fluctuó entre 232\% (Asia) y 350\% (América Latina y el Caribe), se acercó a 179\% en Europa, a 134\% en Oceanía y llegó a $110 \%$ en Norteamérica (Internet World Stats, 2006). Desde luego que estos índices, por llamativos que parezcan, no expresan la realidad en cuanto a las disparidades y la penetración del medio en cada una de esas regiones y entre los diferentes países.

Nadie ignora que la vertiginosa expansión de la red es, y ha sido, desigual tanto si el parámetro es la comparación entre países como si 
el foco de atención es el interior de los mismos. Discontinuamente, internet, va ganando más terreno en determinados, escenarios sociales -los más favorecidos en nivel de desarrollo-, frente a otros de menor estatus socioeconómico. La experiencia digital de los países ricos es sustancialmente diferente a la de las regiones marginadas del sistema mundial. Estados Unidos constituye un caso paradigmático aunque no exclusivo. Por ejemplo, se estima que en 2005 la densidad de usuarios de internet, por cada 100 habitantes estadounidenses, llegó a 63. Una cifra prácticamente igualada por el Reino Unido (62.8\%) y Finlandia (63\%) y sólo superada por Suecia (75.4), Corea del Sur (68.35), Australia (70.4) e Islandia (87). La otra cara de la moneda se refleja en el continente africano. La densidad promedio de internautas en África -considerando a todos los países del continente- apenas alcanzó los 3.75 usuarios por cada 100 habitantes (ITU, 2005).

El acceso diferenciado a las NTIC, dentro de las cuales destaca la red de redes, ha llevado a poner en el centro del análisis los potenciales efectos de estas disparidades para el futuro de muchas sociedades $\mathrm{y}$, dentro de ellas, de no pocos grupos sociales. Tales preocupaciones han dado pie a hipótesis sobre las crecientes brechas informativas y de conocimiento que podrían estar gestándose con el advenimiento de la sociedad de la información y, consecuentemente, de las nuevas formas de diferenciación y desigualdad social (Norris, 2001).

La expansión de la red, al igual que los diversos usos y prácticas que se realizan en torno a ella, están también dando pie a toda una serie de reflexiones y estudios sobre el presente y futuro de las "audiencias" en la sociedad digitalizada (Livingstone, 2004; Neuman, 2002), sobre las aplicaciones de internet, las apropiaciones de la misma, y los efectos sociales e individuales que se están generando (Nie y Erbring, 2002; Castells, 2003). Los análisis manifiestan las preocupaciones de las empresas tradicionales de comunicación, lo mismo que de los especialistas en mercadotecnia, de actores políticos, de comunidades académicas y, desde luego, de quienes tejen cotidianamente la red.

El tipo de estudios, su sofisticación y profundidad, ha estado condicionado no sólo por los intereses y objetivos que subyacen a los patrocinadores sino también, y de manera determinante, por el contexto social y mediático en que se realizan. No es extraño observar que en 
el mundo de alto desarrollo el análisis camina en función de una muy acelerada convergencia mediática y tecnológica (Pew Research Center, 2005). Los usos y apropiaciones de internet están crecientemente ligados, por ejemplo, a los usos de la telefonía celular, o al acceso a la banda ancha o a la tecnología inalámbrica. Los estudios enfatizan el posible desplazamiento de la atención de las audiencias de los medios tradicionales en favor de internet, al igual que la incorporación de esos medios en el mundo de la red y el potencial comercial de esta (European Internet Advertising Association -EIAA, 2005). Consideran la velocidad de adopción de las NTIC a la vida cotidiana y sopesan el rumbo que asumirán en el futuro cercano las actividades laborales, la educación, el consumo, el entretenimiento, la participación ciudadana en la cosa pública, el ejercicio del poder político, las formas de cohesión social, etcétera.

Y si bien esas mismas preocupaciones se reflejan en el mundo de menor desarrollo socioeconómico, las propias condiciones "objetivas" de estas realidades obligan a plantear preguntas similares pero con énfasis distintos. Un tejido menos elaborado de la red, la escasez de infraestructura de telecomunicaciones, el particular manejo del mercado mediático, las condiciones culturales y educativas, la inconsistencia de las políticas públicas en la materia y la misma carencia de recursos para emprender indagaciones rigurosas, operan como factores condicionantes del tipo y nivel de análisis que sobre internet se están llevando a cabo fuera de los países de alto desarrollo.

No obstante, hay interrogantes que atraviesan indiscriminadamente a unos contextos tanto como a otros, y entre ellas se encuentra la evaluación de la presencia de internet y de los usos sociales que se están dando a este medio de comunicación, al igual que las consecuencias que podrían desprenderse de esta realidad.

\section{MÉXICO: UNA DIGITALIZACIÓN EMERGENTE}

En manera alguna la incorporación de México al mundo digital, en particular al desarrollo de internet, puede considerarse tardío. Desde fines de la década de los ochenta se iniciaron los esfuerzos por adoptar esta tecnología de comunicación al mundo universitario, siguiendo en buena 
medida los pasos de su desarrollo en Estados Unidos. De acuerdo con Islas y Gutiérrez (2003)

entre 1989 y 1993, las universidades operaron como únicas proveedoras de acceso a Internet en México...la mayoría de los usurarios de la gran red eran académicos e investigadores adscritos a las principales instituciones de educación superior del país.

Frente a una casi nula política gubernamental en la materia y una exigua participación empresarial en el medio emergente, las universidades mexicanas representaron el espacio innovador desde donde se comenzó a tejer una red digital que tres lustros después alcanza a casi una quinta parte de la población, y en la que la concurrencia de prestadores públicos y privados del servicio también se ha multiplicado.

La expansión de Internet, en México, ha seguido un proceso relativamente "natural", y su penetración ha florecido en los núcleos urbanos y entre los segmentos de población cuyo perfil sociodemográfico se ajusta a las características que, en su momento, sirvieron como campo de cultivo del nuevo medio en otras latitudes. El grueso de la población de internautas en México está dibujado por jóvenes y no tan jóvenes, cuyo nivel de educación es superior al promedio nacional, ajeno al mundo de la pobreza y perteneciente, con una ligera mayoría, al género masculino (AMIPCI, 2005 y 2006).

A partir de que en la segunda mitad de los años ochenta y al comienzo de los noventa, la Universidad Nacional Autónoma de México y el Tecnológico de Monterrey (campus Monterrey), y posteriormente la Universidad de Guadalajara, la de Las Américas, en Puebla, y el Instituto Tecnológico y de Estudios Superiores de Occidente (ITESO) - entre otras pocas universidades- arrancaran el experimento de internet, la línea de prestadores del servicio y de dominios, así como la de usuarios, siguió una tendencia ascendente. Al iniciar los años noventa el número de dominios . $m x$ apenas sumaba 45 , de los cuales 40 eran académicos y cinco eran comerciales. Para 1998 el número de dominios registrados alcanzó los diez mil y en el año 2002 la cifra llegó a los 75 mil, entre los que la mayoría abrumadora se ubicó en la subcategoría de los .com. $m x$. En la actualidad, el total de dominios . $m x$ registrados en todas sus 
subcategorías (.net, .org, .edu, .gob, .com) rebasa los 187 mil (Network Information Center-México, 2006).

Por su parte, los usuarios se están multiplicando. Se estima que en el año 2000 la densidad de usuarios de la red, por cada 100 habitantes, en México rondaba los 5.1 Dos años más tarde el índice prácticamente se duplicó (9.8 internautas por cada 100 habitantes). Para 2005 esa proporción de usuarios aumentó a 17.4 y se calcula que en este año pueda alcanzar los 20 internautas por cada 100 habitantes. De acuerdo con la AMIPCI el número total de usuarios en el país, en el primer semestre del año 2006, se aproximaba a los 20 millones 200 mil habitantes (AMIPCI, 2006).

Algo similar puede decirse sobre el creciente acceso de la población a las computadoras personales. Mientras que en 1999 la proporción de PC por cada 100 habitantes se ubicaba en las 4.4, seis años después, en 2005, la proporción se había triplicado hasta alcanzar las 13.08 computadoras personales por cada 100 habitantes. Actualmente hay en el país $12.5 \mathrm{mi}-$ llones de PC, $60 \%$ de las cuales están en los hogares y de estas $60 \%$ están conectadas a la red (idem, ITU, 2005)

A pesar del dinámico crecimiento de las NTIC en México, comparativamente hablando, los avances relativos a la digitalización de la sociedad -al menos hasta hace poco tiempo- mostraban señales de insuficiencia. Al iniciar esta década, México se ubicaba en el lugar 24 de un grupo de 27 países seleccionados entre Europa, América y Oceanía en cuanto a la densidad de internautas y en un lugar similar en relación con la presencia de las computadoras personales y de la telefonía móvil. En el 2002 el país se encontraba en los últimos lugares entre los países calificados de acceso medio alto de acuerdo con el Índice de Acceso Digital (DAI), desarrollado por la Unión Internacional de las Telecomunicaciones. Por debajo de España, Grecia, República Checa, Chile, Costa Rica y Uruguay. De hecho, para ese año, México no aparecía entre los diez primeros países con mayor DAI en el continente americano (ITU, 2003). Respecto a sus principales socios comerciales (Estados Unidos y Canadá) la desproporción imperante es radical, de más de tres a uno en el porcentaje de la población que tiene acceso a internet, y aún mayor en cuanto a la tasa de PC por cada 100 habitantes.

Con todo, las diferencias entre México y ciertos países latinoamericanos parecen atenuarse paulatinamente. En cuanto a penetración de la red, 
México está a la par de Argentina, ligeramente por debajo de Chile y arriba de Brasil, Colombia, Perú y Venezuela. Después de Brasil, México es el país con mayor número de $\mathrm{PC}$ en Lationamérica, y por lo que respecta a la proporción de estas por cada 100 habitantes, se encuentra en el rango alto del continente latinoamericano (ITU, 2005). Una situación similar impera en el renglón de la telefonía móvil, donde la cobertura se aproxima a $50 \%$ de la población con más de 53 millones de teléfonos celulares en operación (AMIPCI, 2006).

\section{UN OBJETO DE ESTUDIO EN ASCENSO}

A la par de la penetración de las NTIC en el contexto mexicano, ha crecido el interés por estudiar las potencialidades, los usos y las consecuencias positivas y negativas de su desarrollo. Si bien la preocupación está lejos de ser exclusiva del mundo académico, este sirve como termómetro para sopesar la centralidad que gradualmente va adquiriendo internet como medio de comunicación.

Con el paso del tiempo son más y más los investigadores de la comunicación que concentran sus miradas sobre el fenómeno de la red. Los estudios en relación con esta se multiplican en México desde los años noventa, aunque todavía son escasos aquellos que aportan resultados de investigaciones aplicadas o empíricas. En general el conocimiento que circula en revistas especializadas y en los espacios académicos remite a ensayos de análisis y reflexión sobre las consecuencias previsibles y globales de un medio que está trastocando todo tipo de prácticas comunicativas e informativas.

De entre los trabajos académicos que en su momento permitieron situar la problemática del medio y su presencia en la región latinoamericana, no se puede ignorar el ensayo de Raúl Trejo Delarbre sobre lo que bautizó como "la nueva alfombra mágica” (1996), y trabajos posteriores de este investigador y periodista mexicano (por ejemplo, Trejo Delarbre, 1999, 2003 y 2006). En el mismo sentido se encuentra el esfuerzo de Octavio Islas y Fernando Gutiérrez (2000), quienes a fines de los noventa lograron conjuntar a un puñado de especialistas nacionales para debatir los dilemas del "medio inteligente" y sus repercusiones en la mercadotécnica, la seguridad, la prensa, la televisión, la radio, el 
desarrollo de las comunidades virtuales, el papel del Estado, los derechos en la sociedad de la información, etcétera. Cabe señalar que desde el Proyecto Internet del Tecnológico de Monterrey (campus Estado de México), Islas y Gutiérrez han edificado un importante espacio de análisis y reflexión sobre el impacto de la red en el contexto mexicano.

Junto a estos trabajos que podríamos considerar "seminales" en el contexto académico mexicano, algunos otros, también surgidos de la organización de seminarios ex profeso, han planteado agendas pertinentes no sólo para la academia sino para el desarrollo de políticas públicas y sociales frente a internet (cfr. Villanueva, 2000; Carpizo y Carbonell, 2000; Islas, et al., 2002; Solís, 2000 y 2003; Crovi, 2004; Crovi y Hernández, 2004). En el panorama de la investigación sobre los usos de internet en México, no han faltado tampoco las referencias obligadas a experiencias "innovadoras" en materia informativa, como la utilización de la red por parte de la guerrilla neozapatista en Chiapas, desde su irrupción en 1994 que, al margen de cualquier otra consideración, estableció una práctica original y de vanguardia en el campo de la política simbólica generada desde los movimientos contestatarios y rebeldes (Aczel,1997; Martin, 1998; Strikovsky, 2000; Galindo, 1997).

En el todavía limitado cúmulo de investigaciones que focalizan su atención en este objeto emergente de la comunicación, son relativamente escasas las indagaciones que pretenden explorar a través de estudios empíricos y de campo (independientemente de las aproximaciones metodológicas empleadas) los usos y apropiaciones de la red por parte de los internautas mexicanos. En su mayoría, los análisis de esta naturaleza se circunscriben a tesis recepcionales de posgrado o a estudios todavía incipientes, cuya pretensión ha sido explorar con más detalle las formas de utilización de internet en espacios y circunstancias específicas, como las que corresponden a comunidades virtuales, a grupos generacionales, a entornos culturales y educacionales determinados (Keizer, 2000; Garza Martínez, 2000; Winocur, 2004; Cortázar Rodríguez, 1998 y 2004).

Como sea, el perfil de los internautas mexicanos está en proceso de delinearse, sobre todo si el referente son las realidades regionales y locales del país. Hasta donde es posible constatar, son todavía muy escasos los estudios de dominio público que dibujan las características 
de los usuarios de la red, así como los usos más frecuentes y mucho menos las motivaciones y gratificación que de ello se derivan. Quizá el esfuerzo más llamativo al respecto es el que ha venido realizando, en tiempo reciente, la Asociación Mexicana de Internet (AMIPCI), con sus estudios anuales sobre los "hábitos de los usuarios de internet". Sustentado en fuentes directas e indirectas y sin explicitar en detalle su metodología, la AMIPCI ha plasmado un panorama general de los usuarios de la red en el país. Su más reciente informe habla de la existencia de poco más de 20 millones de internatuas mayores de 13 años, de los cuales $90 \%$ radica en zonas urbanas. El reporte sostiene que la penetración de la red ha llegado a $21 \%$ en términos generales y que la tasa se eleva al $33 \%$ en las zonas urbanas.

De acuerdo con el informe 2006 de AMIPCI, en el acceso a la red predomina el género masculino (58\%) y el grupo de edad más joven de los ahí considerados (12 a 19 años) que constituye 39\% de los internautas. Más aún, se afirma que casi $60 \%$ de los usuarios se localizan en el rango de los 12 a los 24 años de edad. De igual forma se establece que la correlación entre ingreso y acceso es evidente. Tras dividir a la población en siete estratos socioeconómicos, el informe señala que prácticamente $40 \%$ de los usuarios de la red está ubicado en los tres niveles superiores y que en las zonas urbanas esa proporción alcanza 52\%.

Sin duda, los reportes anuales de AMIPCI se están convirtiendo en un importante referente para quienes desde muy distintas ópticas e intereses desean explorar las bondades de la red para múltiples fines (comerciales, políticos y educativos). El internauta mexicano es cada vez más el objeto de los apetitos de quienes desean hacer llegar su mensaje a través de internet, aun en situaciones cuestionables. ${ }^{2}$

En suma, si juzgamos por la cantidad de estudios de acceso público podemos concluir que la red de redes y sus usuarios en México

2 Como muestra un botón: se calcula que durante las recientes elecciones federales más de siete millones de correos difamatorios contra el candidato de la izquierda (Andrés Manuel López Obrador) fueron emitidos desde las oficinas y dependencias del Poder Ejecutivo federal. Así pues, en la guerra sucia que distinguió a esa contienda electoral, internet también jugó su papel. 
conforman todavía una zona relativamente inexplorada para publicistas, mercadólogos, estrategas del poder y analistas académicos. Los internautas y sus prácticas de navegación y apropiación de mensajes son un objeto de estudio que recién comienza a construirse, aunque su presencia en la realidad mediática del país es ya incuestionable.

\section{USOS Y USUARIOS \\ DE INTERNET EN JALISCO}

Este trabajo reporta una parte de los resultados de una encuesta sobre consumo mediático aplicada en los primeros meses del 2006 en el estado de Jalisco, región ubicada en el occidente de México (Arredondo, 2006). El análisis de los usuarios y de los usos de internet se elaboró considerando y contrastando los patrones generales de acceso a otros medios de comunicación (televisión, radio, prensa, cine, etc.) Entre las características de la encuesta cabe señalar el hecho de que esta se levantó en toda la entidad de manera tal que el lugar de residencia (zona metropolitana de la capital versus resto del estado) se conformó en una de las variables control, junto con el género y el rango de edad. ${ }^{3}$

¿Qué es lo que se puede decir de los internautas y de la red en Jalisco? ¿Cómo se caracterizan y con qué fines acceden a la red de redes? ¿Cómo está afectando el uso de internet a otros medios de comunicación? Presentamos aquí una sintética respuesta a estas interrogantes.

1. En primer lugar es necesario señalar que a pesar del crecimiento vertiginoso de la telaraña en el país, y en consecuencia de los usuarios de la misma, internet todavía se mantiene como un medio "relativamente marginal" en la dieta mediática de los habitantes de esta región (cfr. cuadro 1). Si bien casi una tercera parte de los habitantes tienen acceso

3 Nota metodológica. El universo del estudio fue la población de 16 años en adelante, residente en el estado de Jalisco. El tamaño de la muestra fueron 957 casos, con representatividad en la ZMG y en el resto del estado. La confiabilidad estadística fue de $95 \%$ y el error estadístico de más/menos $5 \%$. La técnica de muestreo fue aleatorio estratificado polietápico. La encuesta se levantó en 46 municipios, en domicilio con entrevista cara a cara. La fecha de levantamiento fue en los meses de febrero y marzo de 2006. 


\section{CUADRO 1}

CONSUMO MEDIÁTICO EN JALISCO (2006)

Ve televisión

Todos los días

Escucha radio

Todos los días

Lee periódicos

Todos los días

Utiliza internet

Todos los dias
$93.5 \%$

78.7

$66.5 \%$

70.3

$32.6 \%$

16.0

$29.3 \%$

26.4

$\mathrm{n}=957$

a la red (lo que supera el promedio nacional), apenas $11.6 \%$ de los sujetos considerados en este estudio afirma contar con tal servicio en el hogar. Vale la pena señalar, además, que sólo dos terceras partes de los internautas declara poseer una PC en el hogar.

En contraste, prácticamente la totalidad de los entrevistados (98.7\%) afirma tener aparato de televisión en el hogar. La presencia de la televisión en el estado es abrumadora frente a los demás medios de comunicación. $\mathrm{Su}$ incuestionable hegemonía se ha corroborado a través de un simple dato: de acuerdo con los resultados del conteo de población y vivienda del 2005 (una especie de censo intermedio), en Jalisco, al igual que en otras tantas entidades del país, suman más las viviendas que cuentan con televisión que aquellas en las que hay refrigerador (INEGI, 2006).

Como quiera que sea, la presencia de internet ha crecido en forma nada desdeñable. Entre el 2003 y el 2006, el porcentaje de internautas en el estado aumentó aproximadamente seis puntos porcentuales (Arredondo, 2003 y 2006). A comienzos de este último año una tercera parte (29.3\%) de los entrevistados afirmó ser usuario de la red, pero sólo una cuarta parte de los navegantes (26.4\%) se declaró usuario de todos los días, mientras que otro tanto (25\%) se manifestó como usuario de tres a seis días por semana. 
2. El género sí hace cierta diferencia: ciñéndose a la tendencia nacional, la mayoría de los usuarios de la red en Jalisco pertenecen al género masculino (51.8), aunque la desventaja del género femenino es menor en nuestro caso que en el reportado por el estudio de AMIPCI a nivel nacional para el año 2006 (47.8\% en Jalisco versus 42\% en promedio en el país). De hecho se puede decir que en Jalisco, la proporción de internautas es inversa a la distribución de la población general según el género. Es posible suponer que la brecha de acceso por género tenderá a atenuarse aún más con el tiempo, tal y como al parecer ya sucede en otros escenarios sociales.

3. El lugar de residencia también discrimina: considerando la ubicación geográfica de los usuarios de internet, se observa que casi dos terceras partes de los mismos (60.7) se localizan en la zona metropolitana de Guadalajara (ZMG), es decir, en la gran mancha urbana de la capital del estado. Se confirma, pues, que el acceso a la red todavía está fuertemente relacionado con la condición urbana y con un relativo centralismo de los servicios de comunicación y de otra naturaleza que impera en la entidad (con excepción de la televisión, cuya penetración no discrimina lugares). Analizando por separado a la ZMG y al resto del estado, la encuesta arroja los siguientes resultados: en la ZMG la proporción de internautas alcanza $34.3 \%$ de la población total, mientras que en el resto de la entidad los usuarios rondan $23.8 \%$ del total.

4. Un fenómeno primordialmente juvenil: en cuanto a la edad de los internautas en Jalisco, los resultados confirman el patrón general (podría decirse casi universal): el grueso de los usuarios son hombres y mujeres ubicados en los rangos juveniles de la población. La tendencia sigue la línea de una pirámide invertida. Poco más de $50 \%$ de los usuarios considerados se ubica por debajo de los 25 años (cfr. cuadro 2). Se trata de una tendencia que parece repetirse con sus matices en todas las sociedades del orbe.

5. Tres variables importantes: aun cuando el estudio no controló estadísticamente las variables de "educación”, "ocupación” y “educación del jefe de familia”, sí se registraron tales datos. Las tendencias son ilustrativas y consistentes con otros análisis. La mayor proporción de usuarios declara contar con educación media superior (35.7\%), le sigue el grupo de quienes han completado la educación secundaria (29.6) y el tercer sitio 


\section{CUADRO 2}

EDAD DE LOS INTERNAUTAS

$\begin{array}{cc}\text { Años } & \text { Porcentaje } \\ 16 \text { a } 24 & 56.1 \\ 25 \text { a } 34 & 25.0 \\ 35 \text { a } 44 & 10.0 \\ 45 \text { a } 54 & 5.7 \\ 55 \text { a } 64 & 2.5 \\ 65 \text { ó más } & 0.7 \\ & \mathrm{n}=280\end{array}$

lo ocupan quienes cuentan con nivel de educación superior o licenciatura. En cuanto a la "educación del jefe de familia" de los internautas, los resultados indican que la tendencia se inclina hacia el grupo de quienes tienen nivel de educación superior (24.3) seguidos por quienes cuentan con educación media superior (21.4) y educación secundaria (19.6). Finalmente la variable "ocupación" del entrevistado indica que 35\% de los usuarios de internet son estudiantes en activo y $30.7 \%$ empelados (sin especificar más). Sólo $11.4 \%$ declara ser ama de casa y 10.4\% sostiene dedicarse al comercio.

6. Internet: ¿un uso menos intensivo? Quienes navegan en la red, entre semana, lo hacen en promedio 2 horas con 17 minutos al día. Comparados con los televidentes (93.5) y con los radioescuchas (66.5), los usuarios de internet tienden a ser menos intensos en su exposición (cfr. cuadro 3) Una posible explicación de este comportamiento radicaría en el hecho de que sólo $30.4 \%$ de los usuarios están conectados desde la propia casa-habitación. El resto, dos terceras partes de los in-

\section{CUADRO 3}

EN UN DÍA NORMAL, ENTRE SEMANA, ¿CUÁNTAS HORAS...?

Ve televisión

Escucha radio

Usa servicio de internet

$\mathrm{n}$ tv $=895$
2:40 hrs

$3: 36 \mathrm{hrs}$

2:17 hrs

$\mathrm{ntv}=895 \quad \mathrm{n}$ radio $=636 \mathrm{n} \quad$ internet $=280$


ternautas, está obligado a utilizar el servicio en lugares públicos (cibercafés, escuelas o casas de amigos). De esta manera, es posible suponer que la intensidad de uso está relacionada con el tipo de acceso a la red. El acceso desde el hogar se correlaciona positivamente con un uso más frecuente (cfr. cuadro 4).

Por otra parte, cabe también señalar que el acceso al servicio de banda ancha y de alta velocidad es relativamente limitado. De acuerdo con los datos arrojados por la encuesta, 48.6\% de los internautas que están conectados desde el hogar tienen acceso a la banda ancha. El resto opera a través de la conexión identificada como dial-up.

7. La escuela, un territorio cuestionable. El lugar donde más se consulta la red son los cibercafés $(45.7 \%)$. Le sigue el propio domicilio de los usuarios $(27.5 \%)$ y el trabajo (15\%). Sin duda lo más significativo de estos resultados radica en el hecho de que aun cuando la mayoría de los internautas son jóvenes en edad de estudiar, la escuela represente el espacio en donde menos se utiliza el servicio de internet. Quizá esto se explique por la carencia del servicio en las escuelas (lo que hablaría de serias deficiencias en la infraestructura digital del sistema educativo), o tal vez por que la "cultura de la cibernavegación" tiene una mayor carga lúdica en lugares ajenos al sistema de educación formal. Un tema para investigar.

8. Internautas epistolares. Respecto a los principales usos que se le dan a internet, los cibernautas de Jalisco parecen inclinarse fuertemente

\begin{tabular}{|c|c|c|}
\hline \multicolumn{3}{|c|}{$\begin{array}{c}\text { CUADRO } 4 \\
\text { EN EL TRANSCURSO DEL DÍA DE AYER, } \\
\text { ¿CUÁNTAS HORAS USÓ INTERNET? }\end{array}$} \\
\hline & Tiene servicio en casa & No tiene servicio en casa \\
\hline No utilizó & $30.6 \%$ & $58.5 \%$ \\
\hline Menos de1hr. & $10.6 \%$ & $8.2 \%$ \\
\hline 1 a 3 hrs. & $45.9 \%$ & $29.7 \%$ \\
\hline 3 a 6 hrs. & $11.8 \%$ & $2.1 \%$ \\
\hline 6 a 9 hrs. & ------ & $1.0 \%$ \\
\hline 9 a 12 hrs. & $1.2 \%$ & $0.5 \%$ \\
\hline Total usuarios & 85 & 195 \\
\hline
\end{tabular}


por la comunicación epistolar (42\% sumando el correo electrónico y el chat) y por la obtención de información con fines educativos y laborales. En una posición más rezagada se localiza la descarga de contenidos musicales y la búsqueda de información noticiosa. Y en la zona abiertamente marginal se ubican usos tales como los comerciales, los de servicios gubernamentales y los que tienen que ver con el manejo de imágenes en movimiento (cfr. cuadro 5)

9. La edad hace cierta diferencia: este patrón de usos sufre variaciones si se analiza a la luz de otras variables. Así, por ejemplo, entre los jóvenes de 16 a 24 años es más clara la tendencia a utilizar los "chat rooms" que en cualquiera otro de los rangos de edad contemplados por la encuesta. Lo mismo sucede si el uso se refiere a "descargar música". En cambio si el caso remite a la utilización del "correo electrónico" la tendencia se inclina hacia el grupo de 25 a 34 años. Los cibernautas que oscilan entre los 45 y los 65 años se cargan más que el resto hacia la búsqueda de noticias en la red. En tanto, los internautas del rango de los 35 a los 44 años tienden a bajar documentos e información en una mayor proporción que los jóvenes de 16 a 24 años y que los adultos jóvenes de 25 a 34 años.

\section{CUADRO 5}

¿QUÉ SERVICIO DE INTERNET USA MÁS FRECUENTEMENTE?

Descargar documentos de apoyo

para el trabajo o la escuela

$23.2 \%$

Comunicarse por correo

$21.0 \%$

Chatear

$21.0 \%$

Descargar música

$10.5 \%$

Buscar información noticiosa

$10.5 \%$

Consultar servicios de gobierno

Utilizar juegos en línea

Comprar y/o vender

Hacer transacciones bancarias

Descargar películas y videos

Descargar software

Pagar servicios (teléfono, luz, agua, etc) 
Por otra parte, si la variable a través de la cual se observan las tendencias de uso es el género, el resultado es una variación menor. Ligeras diferencias se manifiestan, por ejemplo, en cuanto a la práctica del "chateo" -a la que las mujeres son más adeptas-, o en cuanto al uso del correo electrónico -al que los varones parecen preferir. De todos los usos relevantes de internet, la mayor diferencia entre hombres y mujeres parece radicar en el concerniente a la "descarga de documentos para el trabajo o la escuela". Ahí las mujeres se aplican ligeramente más que su contraparte de género.

En contraste con lo que sucede con los usos predominantes de otros medios, como la televisión o el consumo de impresos, la diferencia de género todavía no se percibe radical entre los internautas de Jalisco. Aquí no sucede lo mismo que con la televisión, donde la preferencia por las telenovelas, por los noticiarios y por los programas deportivos discrimina claramente a las mujeres de los hombres.

Como ya se sugirió, el acceso desde la propia casa habitación constituye un patrón de diferenciación en cuanto a la intensidad con que se utiliza la red. Sin embargo, en el terreno de las aplicaciones o usos predominantes de la red, este tipo de acceso no parece dar cuenta de variaciones significativas más que en tres casos: a) la descarga de documentos, y b) la práctica de comunicarse utilizando el "chat room". Ambas a favor de quienes sí están conectados a internet desde el hogar. La otra diferencia significativa, c) se localiza en la "búsqueda de noticias", a la que son más adeptos los internautas sin servicio en la casa habitación.

\section{PATRONES DIFERENCIADOS DE CONSUMO MEDIÁTICO: INTERNAUTAS VERSUS NO-INTERNAUTAS}

Entre las conjeturas que han surgido en torno a la utilización de la red y sus efectos, sobresale una en particular que establece una diferenciación de los patrones en el uso de otros medios de comunicación por parte de quienes tienen acceso a internet. Un análisis comparativo entre los internautas identificados en este estudio y quienes declararon no acceder a la red de redes en Jalisco, arroja resultados sugerentes que en parte sostienen y en parte contradicen la creencia de que los usuarios de la red tienen comportamientos distintos mediáticamente hablando. 
En términos generales, los resultados muestran que quienes acceden a internet (por lo menos en un estadio similar al que impera actualmente en México, y en particular en Jalisco) cuentan con mayor acceso a los diversos "recursos de comunicación" que la contraparte de la población no incorporada a la cibercultura. Esta es una breve descripción de tales hallazgos (cfr. cuadro 6).

1. Televisión e internautas. En lo que respecta a la tenencia de televisión, y dado su carácter prácticamente universal, no existe una diferencia entre internautas y no-internautas en Jalisco. Sin embargo, si el criterio es

\section{CUADRO 6 \\ ACCESO MEDIÁTICO DIFERENCIADO EN JALISCO}

\begin{tabular}{lcc} 
& Internautas & No-internautas \\
Tiene televisión en casa & 99.6 & 98.4 \\
Tiene televisión de paga & 55.7 & 32.5 \\
Ve televisión & 96.8 & 92.2 \\
Ve televisión (1 a 3 hrs diarias) & 69.7 & 67.3 \\
Tiene aparato de radio & 94.3 & 87.4 \\
Escucha radio & 77.1 & 62.0 \\
Escucha radio (1 a 3 hrs diarias) & 55.1 & 42.1 \\
Lee periódicos & 46.4 & 26.9 \\
Lee periódicos todos los días & 13.1 & 18.1 \\
Lee revistas & 68.9 & 36.5 \\
Lee revistas de moda/belleza & 25.4 & 19.0 \\
Lee revistas científicas & 11.4 & 2.1 \\
Lee libros & 72.9 & 41.4 \\
Leyó en los últimos tres meses & 79.9 & 79.9 \\
Va al cine & 62.9 & 16.7 \\
Va una vez a la semana & 13.1 & 6.2 \\
Tiene videocasetera & 64.6 & 49.8 \\
Cuenta con DVD & 85.4 & 62.3 \\
Ve películas en video/DVD & 79.3 & 47.1 \\
Ve películas mexicanas & 17.6 & 28.5 \\
Ve una o dos películas por semana & 56.3 & 48.9 \\
Tiene computadora en casa & 60.4 & 20.5 \\
\hline
\end{tabular}


el acceso al servicio de "televisión de paga", entonces sí se manifiesta una clara diferencia. Los usuarios de la red cuentan en mayor proporción que los no-usuarios con tal tipo de servicio (cable, Sky y otras modalidades). Además, los internautas se muestran ligeramente más propensos a ver la televisión que los no usuarios de la red. Pero tal diferencia seguramente no pasaría una prueba estadística. En el mismo sentido, los usuarios de internet que declaran "no ver televisión", son menos que los no-usuarios que declaran lo mismo. Prácticamente no hay mayores diferencias en la frecuencia con que unos y otros afirman exponerse a la televisión. No obstante, puede decirse que en su calidad de televidentes, los internautas son menos proclives a las telenovelas y a los noticiarios, pero se inclinan más por los documentales, las series, las películas y los programas de comedia. Posiblemente todo ello se explique por la variable "acceso a la televisión de paga" y por el mayor abanico de opción que presumiblemente ofrece esa modalidad televisiva.

2. Radio e internautas. Se observa una ligera diferencia entre los internautas y su contraparte, por lo que respecta a la tenencia/acceso a la radio. Esa diferencia se ensancha al considerar la práctica cotidiana de ser radioescucha, una tendencia que se observa marcadamente entre los usuarios de la red, quienes se exponen más a la radio. Con todo, la frecuencia con la que se exponen unos y otros al tradicional medio no sufre variaciones considerables. Y esa misma paridad se refleja, por ejemplo, en el relativamente escaso interés de ambos grupos por las noticias radiofónicas.

3. Medios impresos e internautas. Entre los usuarios de la red en Jalisco, la lectura de diarios es abiertamente mayor que entre los no-internautas. La proporción es casi de dos a uno. Sin embargo, los escasos lectores de diarios que se declaran no-internautas mantienen un patrón similar de acceso/frecuencia a este medio que los menos escasos (pero de ninguna manera abundantes) lectores de la prensa que acostumbran navegar en el ciberespacio. En este caso, la lectura de diarios no identificó esa práctica para quienes lo hacen a través de la misma red. Como sea, la condición de internauta está, hasta el momento, abiertamente vinculada con una mayor propensión a la lectura de diarios. Esa misma tendencia se observa al considerar la "revistas" como medio al que acceden unos y otros. Una diferencia considerable se manifiesta entre 
internautas y no-internautas en este renglón. La tendencia se corrobora tanto en el caso de las revistas como en el de la lectura de libros.

Una brecha de más de treinta puntos porcentuales divide a los internautas que se declaran lectores de libros de los no-internautas que abrazan el mismo hábito. Pero a pesar de la gran diferencia, la misma proporción en los dos grupos (79.9\%) sostiene haber leído algún libro por placer personal en los últimos tres meses. En ese caso, como en los abordados con anterioridad, la frecuencia de consumo mediático se asemeja en ambos grupos. La diferencia, pues, radica en el acceso.

4. Cine e internautas. Con una proporción de casi cuatro a uno, los internautas superan a los no-usuarios de la red como cinéfilos. De la misma manera, los internautas que declaran asistir a las salas de cine cada semana, duplican a quienes se manifiestan en el mismo sentido dentro del grupo opuesto. Por lo demás, los gustos de unos y otros, considerando la nacionalidad de las películas, no varía sustancialmente. Por su parte, el acceso/tenencia de reproductores de video y/o DVD es marcadamente distinto entre ambos grupos, siempre a favor de los internautas. Los hábitos también lo son. Quienes se declaran usuarios de internet son más proclives a ver películas a través de estos medios en una proporción muy superior a los no-usuarios, mientras que su preferencia por los filmes mexicanos se discrimina por ese factor. Los internautas gustan menos de los productos fílmicos nacionales que los no-internautas.

\section{UN COMENTARIO FINAL}

Los datos aportados en este trabajo constituyen una aproximación inicial a un fenómeno -el acceso y los usos de internet- que está adquiriendo una relevancia incuestionable en prácticamente todos los contextos sociales del orbe. Los resultados dan cuenta del nivel de penetración de la red en una región específica de México, y en ese sentido pretenden abonar al conocimiento de esta realidad más allá de los datos agregados que dibujan el crecimiento y las prácticas de los internautas en el plano nacional y en el global.

Comparativamente hablando, los resultados corroboran algunas de las tendencias observadas en otras latitudes, pero aportan, simultáneamente, información específica de una población como la que radica en Jalisco. 
Los internautas locales son similares y distintos a los internautas de otros países, y seguramente de otras regiones del mismo país (de los que, por cierto, sabemos poco). Como en casi cualquier parte del mundo, el acceso a la red de redes está correlacionado con el nivel de educación, con la edad, con el lugar de residencia (población rural o urbana), con el nivel socioeconómico y en menor medida con el género. La tendencia, que por lo demás está cambiando en los países más desarrollados, remite a preocupaciones que flotan en el aire desde hace tiempo.

Así pues, en tanto algunas tendencias desiguales se observan en la realidad local, es posible inferir la existencia de "brechas digitales" que deberían analizarse con mayor detenimiento. ¿En qué medida las diferencias de acceso y de uso deben contemplarse como pasajeras e irrelevantes? ¿Son necesarias políticas públicas específicas que atenúen las diferencias, o habrá que esperar a que la dinámica propia de un mercado en franca expansión se haga cargo del asunto? Jalisco es un estado privilegiado en el escenario mexicano, pero sus niveles de desigualdad sin duda tienen correspondencia en el mundo digital. De momento sólo queda reconocer que el acceso a la red es todavía privilegio de una minoría en Jalisco, aun cuando proporcionalmente la entidad está arriba del promedio nacional.

La información presentada nos habla también de una comunidad de internautas cuyos hábitos tiene rasgos distintivos. La red es vista y apropiada, fundamentalmente, como un medio para la relación interpersonal y, en un segundo momento, como apoyo a las tareas educativas y laborales. Los usos de otra naturaleza-como los comerciales- todavía resultan marginales para los internautas de esta región. La búsqueda de información noticiosa apenas ocupa un lugar secundario en el menú de los usuarios. Y qué decir con respecto a aquellos usos que en teoría hablarían de los niveles de participación ciudadana en la cosa pública. El gobierno, sus servicios y la información que circula en la red están lejos de constituir un platillo apetitoso para los internautas jaliscienses. Su consulta es prácticamente nula. Cabría interrogar sobre la causa de tal realidad: ¿falta de interés por la información oficial o falta de credibilidad en esas fuentes?

Otro de los aspectos relevantes de este estudio tiene que ver con los accesos diferenciados en el conjunto de opciones mediáticas para los usuarios y los no-usuarios de la red. De momento se observa una clara tendencia con respecto al mayor potencial de acceso a $-\mathrm{y}$ en algunos 
casos de uso de- los medios de comunicación tradicionales en beneficio de los internautas. ¿Estamos quizá frente a una dinámica que tiende a ensanchar la brecha de oportunidades comunicacionales entre la población? ¿Los de por sí beneficiados tienden a beneficiarse más?

Finalmente, es evidente que los resultados aquí expuestos no sólo obligan a mantener algún tipo de seguimiento sobre las dinámicas en marcha, sino que exigen considerar nuevas aproximaciones al objeto de estudio desde ópticas teóricas y metodológicas diferentes. El estudio de los usos y de las apropiaciones de internet lo mismo requiere de la estrategia de encuestas que de estudios cualitativos más profundos. Aquí sólo se ha esbozado parte de una agenda de investigación a seguir en el futuro.

\section{Bibliografía}

ARREDONDO RAMÍREZ, Pablo (2003) Encuesta Estatal sobre Medios y Audiencias en Jalisco. Reporte de investigación, División de Estudios de la Cultura y Centro de Estudios Estratégicos para el Desarrollo, Guadalajara: UdeG.

- (2006) Encuesta Estatal sobre Medios y Audiencias en Jalisco, reporte preliminar, División de Estudios de la Cultura y Centro de Estudios Estratégicos para el Desarrollo, Guadalajara: UdeG.

ACZEL, Audrey Marianne (1997) A communications analisis of the Chiapas uprising: Marcos publicity camping on the Internet, tesis de maestría en artes, McGill University.

(AMIPCI) ASOCIACIÓN MEXICANA DE INTERNET (2005 y 2006) Hábitos de los usuarios de Internet en México. http://www.amipci.org. mx. 10 de octubre de 2006.

CARPIZO, Jorge y Carbonell, Miguel (coords.) (2000) Derecho a la información y derechos humanos, México: UNAM.

CASTELlS, Manuel (2003) La Galaxia Internet. Reflexiones sobre Internet, empresa y sociedad, Barcelona: Debolsillo.

CORTÁZAR R. Francisco Javier (2004) “Chicanos y mexicoamericanos en tres comunidades electrónicas”, Comunicación y Sociedad, núm. 2, nueva época, Guadalajara: UdeG.

- (1998) "Ciberrelaciones: amistad, amor y sexo en los salones de chat", Comunicación y Sociedad, núm. 34 septiembre-diciembre. Guadalajara: UdeG. 
CROVI, Delia (coord.) (2004) Hacia la sociedad de la infrormación y del conocimiento, Memorias de PANAM II. UNAM-FCPyS, México, D.F.

- y Hernández, Francisco (coords.) (2004) Internet y televisión. Una mirada a la interculturalidad, Guadalajara: UdeG, Ayuntamiento de Zapopan, UAM-Xochimilco.

EUROPEAN INTERNET ADVERTISING ASSOCIATION (EIIA) (2005) Mediascope Europe Study, http:/www.eiaa.net/casestudiespp/

GALINDO C., Jesús (1997) “Comunidad virtual y cibercultura: el caso del EZLN en México”, Estudios de las Culturas Contemporáneas, vol. III, época II, núm. 5, Programa Cultura-Universidad de Colima.

GARZA MARTíNEZ, Guillermo (2000) Estudio de la adopción, características de los usuarios y hábitos de uso de Internet en el área metropolitana de Monterrey, tesis de maestría, ITESM, Campus Monterrey.

INEGI (2006) II Conteo de población y vivienda, 2005, México, D.F.

INTERNET WORLD STATS (2006) Usage and population statistics, 26 de septiembre de 2006, www.internetworldstats.com.

(ITU) INTERNACIONAL TELECOMMUNICATIONS UNION (2005) Basic Indicators, 24 de octubre de 2006, http://www.itu.int/home/

- (2003) "Índice de acceso digital de la UIT: la primera clasificación completa de las TIC en el mundo", comunicado de prensa, http:// www.itu.int/home/

ISLAS, Octavio y Gutiérrez, Fernando (2000) Internet el medio inteligente, México, D.F: ITESM / CECSA,

- et al. (comps.) (2002) Explorando el ciberperiodismo latinoamericano, México, D.F: ITESM / CECSA,

- y Gutiérrez, Fernando (2003) "El medio que cambió a la comunicación”, Revista Mexicana de Comunicación, núm. 83, septiembreoctubre.

KEIZER, Jenka (2000) A study of social and academic uses of the Internet by high school students, tesis de doctorado, University of Alabama.

LIVINGSTONE, Sonia (2004) "The Challenge of Changing Audiences", European Journal of Communication, vol. 19, núm. 1

MARTIN, Brigitte (1998) Le cyber-activisme des Zapatistes, tesis de maestría en artes, Universidad Laval, Canadá. 
NETWORK INFORMATION CENTER NIC-México (2006) http://www. nic.mx

NEUMAN, W. Russell (2002) El futuro de la audiencia masiva, México: Fondo de Cultura Económica.

NIE, H. Norman y Erbring, Lutz (2002) "Internet and mass media: A preliminary report”, IT!\&Society, vol. 1, issue 2, Fall.

NORRIS, Pippa (2001) Digital divide. Civic engagement, information poverty, and the Internet worldwide, Cambridge: Cambridge University Press.

PEW RESEARCH CENTER (2005) "Internet: the mainstreaming of Online Life", Trends 2005, Washington.

SOLÍS LEREE, Beatriz (coord.) (2000) Los medios públicos de comunicación en el marco de la reforma del Estado en México, Cámara de Diputados/FKA/UNESCO/Coneicc/UIA/FELAFACS/UAM/AMEDI.

- (coord.) (2003) El reto de México ante la Cumbre Mundial de la Sociedad de la Información, Fundación Conrad Adenauer, Comisión de CyT Senado de la República, UNESCO, UIA, AMEDI.

STRIKOVSKY, Vestel Sandra (2000) "EZLN: ¿Una guerrilla cibernética?", Espacios de Comunicación, núm. 4. México: UIA,

TREJO DELARBRE, Raúl (1996) La nueva alfombra mágica. Usos y mitos de Internet, la red de redes, México: Fundesco/Diana.

- (1999) "La Internet en América Latina”, en García Canclini y Moneta (coords.) Las industrias culturales en la integración latinoamericana, México: Grijalbo/UNESCO/Sela.

- (2003) "Una red para la gente", en Solís Leree (coord.) El reto de México ante la Cumbre Mundial de la Sociedad de la Información, FKA/ Senado de la República/ UNESCO/UIA/UAM/AMEDI

- (2006) Viviendo en El Aleph. La sociedad de la información y sus laberintos, Barcelona: Gedisa/ILCE.

VILlANUEVA, Ernesto (coord.) (2000) Hacia un nuevo derecho de la información, México: UIA/Fundación Conrad Adenauer.

WINOCUR, Rosalía (2004) "La computadora y el Internet como estrategia de inclusión y recurso de movilidad social en el imaginario de los pobres", Revista Iberoamericana de Comunicación, núm. 7 otoño-invierno. 